\title{
SECURE MICROSERVICES - A REVIEW APPLYING THE THEORY OF THE CONSOLIDATED META-ANALYTIC APPROACH
}

\author{
Phillipy Ferreira ${ }^{1}$, Welyngton Dal Prá ${ }^{1}$, Daniel N. Araújo ${ }^{1}$, Gabriel de O. Alves ${ }^{1}$, \\ Lucas M. C. e Martins ${ }^{1}$, William F. Giozza ${ }^{1,2}$, and Rafael T. de Sousa Jr. ${ }^{1,2}$, \\ ${ }^{I}$ Decision Technologies Laboratory (LATITUDE.UnB) - University of Brasilia \\ ${ }^{2}$ Electrical Engineering Department, University of Brasilia
}

\begin{abstract}
The goal of this paper is to address the relevance of applying microservice based system architecture, with an emphasis on the challenges of implementing security in this model. Based on the impact this brought to the academic literature, an in-depth bibliographic analysis was performed using the Theory of the Consolidated Meta-Analytic Approach to trace inter-relationships between the studies already performed on security in microservices in order to identify trends, main authors, research centers, relevant papers and keywords.. From a base of 837 Scopus papers, some of the main facts found through the analysis of bibliographic metadata were that the authors of greatest influence are M. Fowler, S. Newman, R. Buyya, Y. Chen, P. Jamshidi, and Y. Zhang, the countries that published the most on the subject were China, USA, and Germany and that the subject is still on the rise in academic literature.
\end{abstract}

\section{KEYWORDS}

Microservices, Security, Bibliographical Review, Bibliometrics

\section{INTRODUCTION}

There is a remarkable rise in the study and employment of microservices in system architectures (Kratzke, 2018). Companies like Amazon, Netflix (Newman, 2015), Google, Twitter and Uber with great impact and influence in the technology area adopted this solution (Kratzke, 2018), attracting even more attention on the subject.

Considering the great relevance and predominance of the architectural model of systems based on microservices (Kratzke, 2018), some challenges such as security are imposed (Alshuqayran et al., 2016) in face of the transformation of traditional architecture from complex 
IADIS International Journal on WWW/Internet

monolithic systems to several simple microstructures whose complexity is now transferred to the interconnection and control of these microsystems (Yarygina and Bagge, 2018).

Some research challenges in the literature are communication/integration between services, performance, fault tolerance, security, $\log$ and monitoring, and deployment operations (Alshuqayran et al., 2016).

After carrying out a bibliographical survey on scientific bases such as Scopus, Web of Science (WoS) and Google Scholar, it was found that there is a wide bibliography on the subject, therefore there is a need for a thorough review focused on security, since it is one of the central issues in the literature (Alshuqayran et al., 2016) (Chondamrongkul et al., 2020).

The aim of this study is to perform a literature review in order to consolidate and validate interrelations verified in an in-depth investigation based on bibliometric laws and their analysis, in order to highlight factors, relationships and trends about security applied to microservices. The following research questions guided this study: 1) Which are the main authors related to security in microservices? 2) Which are the most prolific countries in microservice security in the literature? 3) Which are the main research centers, and where are they located? 4) What are the most recurrent keywords about security in microservices?

The contribution of this work is to identify trends and relevant bibliometric information based on an in-depth bibliographic analysis through the Theory of the Consolidated Meta-analytical Approach (TEMAC) (Mariano and Rocha, 2017).

Section 2 presents an introduction to the theme of security of microservices, with history, challenges and approach that will be used in the work. Section 3 discusses the TEMAC methodology that was used in the systematization. Section 4 shows the application of the methodology, subdivided into 4.1 Research Preparation, 4.2 Data presentation and Inter-relation and 4.3 Research Validation. And the conclusion of the paper is in Section 5.

\section{CONTEXT}

Microservices, according to Newman (2015), are small autonomous services working together. The author emphasizes services' autonomy stating a microservice must be an independent process that can be deployed in its own infrastructure separately and independent from other services, regardless of whether the infrastructure is local or over a network. Newman also lists some principles of this model, such as: modeling around concepts or business domains, automation culture, abstraction of internal implementation details, decentralization and autonomy in decision making, independent deployment, fault isolation, and high monitoring capability.

Some of this model's main advantages are scalability, independent deployment, and the ease of maintenance, and also extend the advantages already obtained with the adoption of the models of Service Oriented Architecture (SOA) and Distributed Systems (Newman, 2015) (Kratzke, 2018).

A software is made up of several parts that need to communicate in order to achieve its goal, regardless of the architectural style used. In the case of an application developed in the microservice architecture, its features are decomposed into microservices that are separate and independent from each other (Lewis \& Fowler, 2014). This also implies that all communication between them must take place via the network (Newman, 2015). In this scenario, the concern with security aspects of services is increased. 
In this sense, one of the most relevant themes in the microservices bibliography is the issue of security (Alshuqayran et al., 2016) (Chondamrongkul et al., 2020), since: 1) the granularity of the microservices architecture is composed of services that run on the network; 2) the services usually run in cloud-based containers, so the security of the system is strongly based on the reliability of these containers; 3) the micro-services must trust that the authentication and authorization control will do their job properly; 4) as the services are scattered on the network, the data transfer over the network is vulnerable to attacks (Chondamrongkul et al., 2020).

By comparing some models of bibliographic review such as Qualitative Review, Integrative Review, Systematic Review and Meta-analysis, it was found that meta-analyses and systematic reviews are more limited to quantitative surveys closed in statistical analysis, making it possible to reduce the bias of other studies or even integrate several primary studies, while the other reviews, although they also allow for qualitative analysis, remain in the scope of summarization or review of theories, studies and methods.

Therefore, it was defined to use the Theory of the Consolidated Meta-Analytic Approach (TEMAC), since this method allows adopting qualitative and quantitative approaches (Mariano and Rocha, 2017) utilizing defined and standardized stages for the review and analysis of bibliographic metadata, enabling the identification of trends, areas of focus, cooperation and work relevant to the area in academic literature.

\section{METHODOLOGY}

The meta analytical approach proposed by TEMAC aims to trace correlations and trends in articles through a method designed in stages that can be assisted by tools such as LibbreOffice Calc, VOSviewer, TagCrowd, and Publish or Perish (Perdigão et al., 2019).

The Theory of the Consolidated Meta-Analytic Approach (Mariano and Rocha, 2017) presents 3 stages that must be followed in order to obtain analytical results: Stage 1 - The preparation of the research, delimitation of the scope and selection of the database; Stage 2 - The presentation of the data inter-relations and Stage 3 - Detailing, integrating model and evidence-based validation.

The TEMAC (Mariano and Rocha, 2017) also allows applying concepts of bibliometry, a branch from the Information Science field (Beuren and Souza, 2008) that is based on a set of rules and principles applying mathematical and statistical methods. Some of the main laws and bibliometric principles used in this work were: 1) the Law of Elitism which is determined by $\sqrt{k}$ where $\mathrm{k}$ is the total number of authors in the area, and this calculation would determine the elite of authors in the area; 2) The 80/20 Rule (Pareto principle) to estimate the relevance degree of the keywords, authors, bibliographic coupling, and analysis of co-citations in a given area of knowledge (Guedes and Borschiver, 2005). 


\section{RUNNING THE TEMAC}

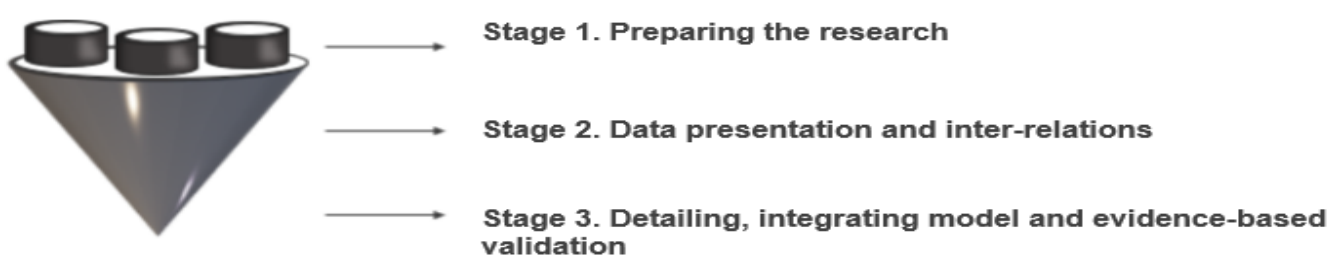

Figure 1. The stages of the TEMAC methodology

Source: adapted from (Mariano and Rocha, 2017)

According to the TEMAC methodology described in Mariano and Rocha (2017), our analysis will be executed in 3 stages: 1) preparation, 2) data presentation, and 3) validation. Figure 1 illustrates this methodology.

\subsection{Preparing the Research}

One of the questions raised by TEMAC (Mariano and Rocha, 2017) is: What is the descriptor, string or keyword of the search? To find the best descriptor the following strategy was used through an algorithm: 1. searching the main terms used in the literature in Scopus, WoS and Google Scholar bases; 2. validation of the main terms used in the most relevant articles; 3. searching with the raised terms and evaluation of the results; 4. incorporation of the terms "AND", "OR" and "NOT" to limit the results found; 5. validation of the results found: if they corresponded to the focus of this review; 6. refinement and execution of step 3 again until the step 5 condition is verified.

After the first evaluation, the research was carried out only with the terms "MICROSERVICE" and "SECURITY". One of the papers returned was the systematic study of Alshuqayran et al. (2016) which lists a set of terms related to security in microservices such as: "secure", "authentication", "authorization", "OAuth", "OAuth2", "encryption", "vulnerability" and "attack". Such terms have been integrated as security synonyms for this search descriptor.

Among the results presented, it was verified through the analysis of the keywords diagram in Fig. 2 that there were many works related to the Internet of Things (IoT), however it was delimited in the scope of this review that the architecture of microservices approached is the conventional structure systems (non-IoT) (Washizaki et al., 2020), thus excluding microservices applied to the Internet of Things, due to the particularities of this model, although an abundant bibliography was observed.

Based on data extracted from the Scopus Platform, Fig. 2 was generated using the TagCrowd tool, with which it can be observed that, excluding the term "microsservice" itself, other keywords such as "cloud", "service", "container"and "virtualization" are frequent in works related to security in microservices. 


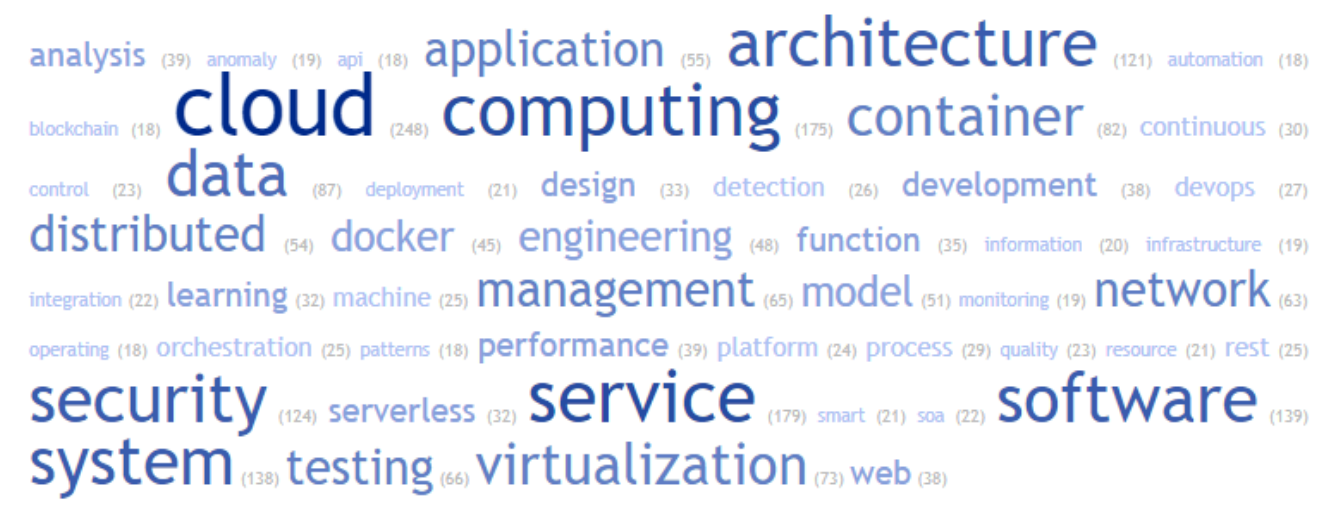

Figure 2. Keyword map

Source: Generated by TagCrowd with data extracted from Scopus

After some repetitions of the steps described, a satisfactory string was found for the scope of this work, and within the comprehensive result found, the presence of several articles with academic relevance and consistent with the objective of this review was confirmed. The search descriptor used in the scientific databases was:

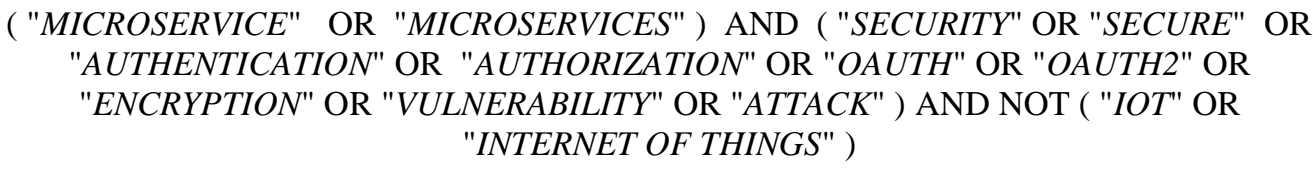

Following the first stage of TEMAC, it is important to delimit the research space-time, therefore the first article found with the term microservice is from 2012, "A semantic metadirectory of services based on web mining techniques" by Fernández-Villamor \& et al, with only 3 citations found, the theme continued with about 2 publications per year, without any impactful publication, until 2015, when the book "Building Microservices: Designing Fine-Grained Systems" by Sam Newman is published. The term microservice gains relevance after this date, proving the great impact of the work. It soon becomes clear that the works with the greatest academic impact begin to be published after 2015, as will be presented later, thus this research was refined to bring results between the years 2015 to 2020 .

Looking at Fig. 3 it is possible to see that the subject is on the rise, presenting an upward curve from 2015 to 2019, and the year 2020 has nearly $96 \%$ of the total number of articles published in 2019 in 11 months, and also considering the impact of the global pandemic which shows that this review is still relevant to the area. 
IADIS International Journal on WWW/Internet

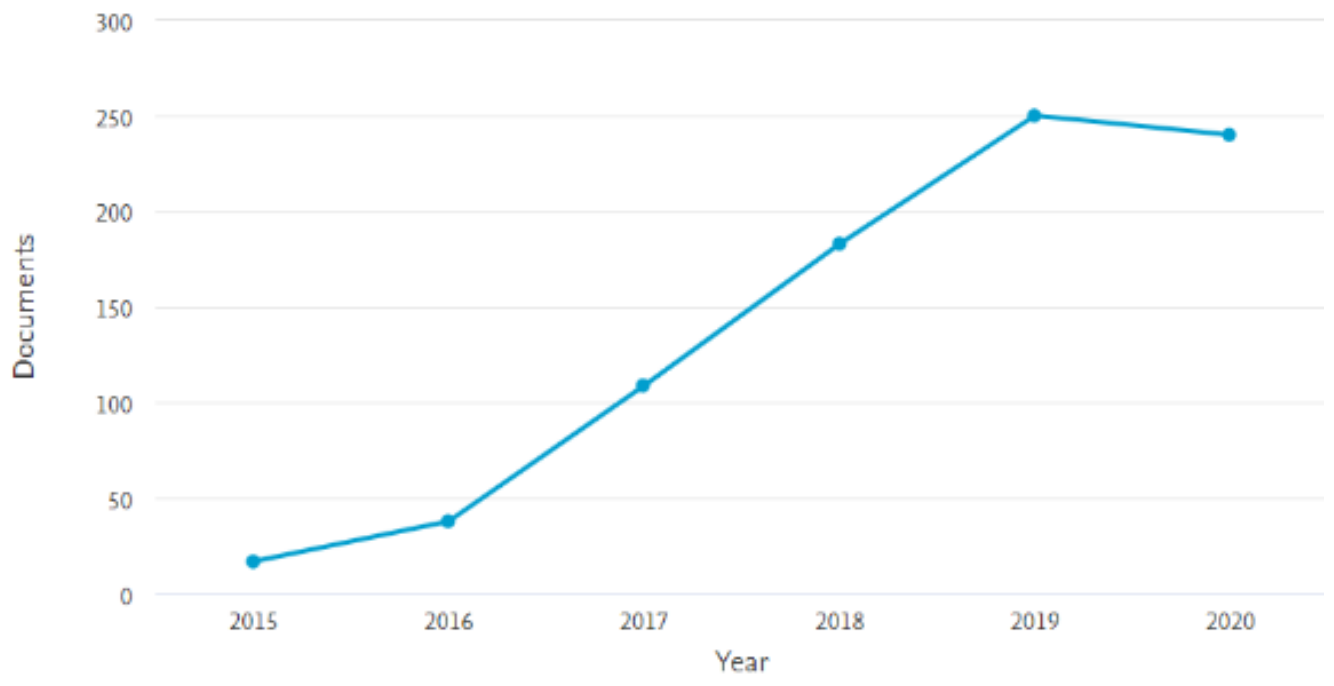

Figure 3. Documents by year Source: Data extracted from Scopus

After analyzing the main scientific databases, such as Web of Science, Scopus, Microsoft Academic and Google Scholar, since the databases have different contents and algorithms (Rovira et al., 2019), it was decided to use only the Scopus platform, mainly because it has better coverage of science and technology journals, covers more global content, about $60 \%$ of the content is from outside the U.S., and has better time coverage of the last 5 years (Mariano and Rocha, 2017).

After analysis carried out at Scopus, the most prominent areas on the subject are Computer Science, Engineering, Mathematics and Decision Sciences as shown in Figure 4. This filter made it possible to restrict the results, since works involving the security theme had been identified as the main subject, such as health services, and used microservices within their articles, which polluted the research base. 


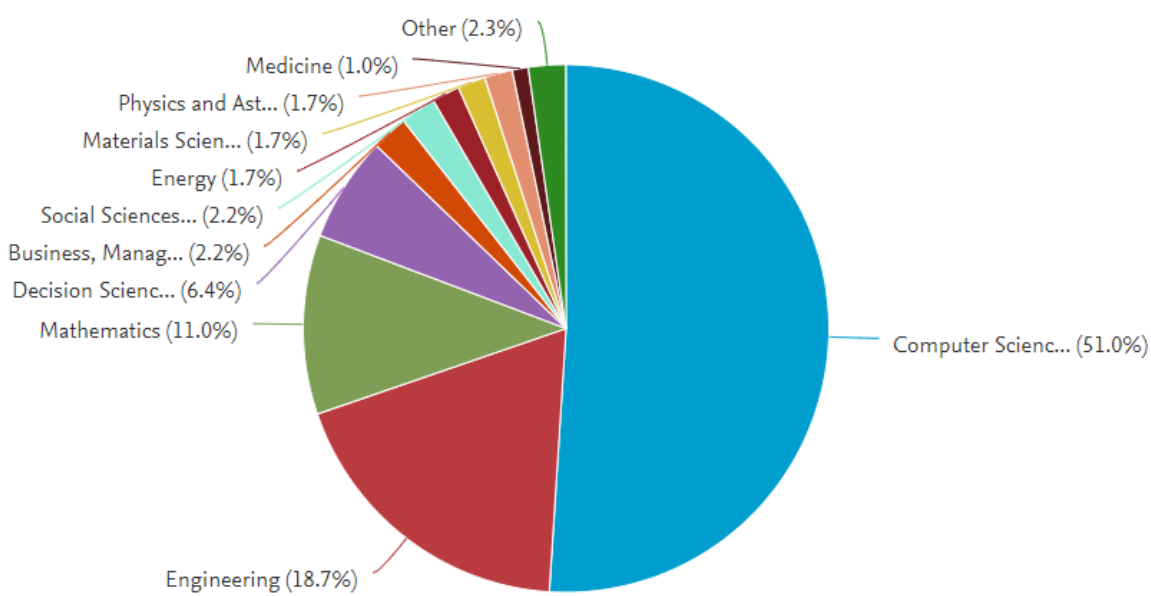

Figure 4. Documents by subject area Source: Data extracted from Scopus

A relevant feature observed in Figure 5 is that most of the documents related to the analyzed topic come from conferences (almost 70\%), highlighting the important role of conferences in the production of scientific knowledge related to the subject.

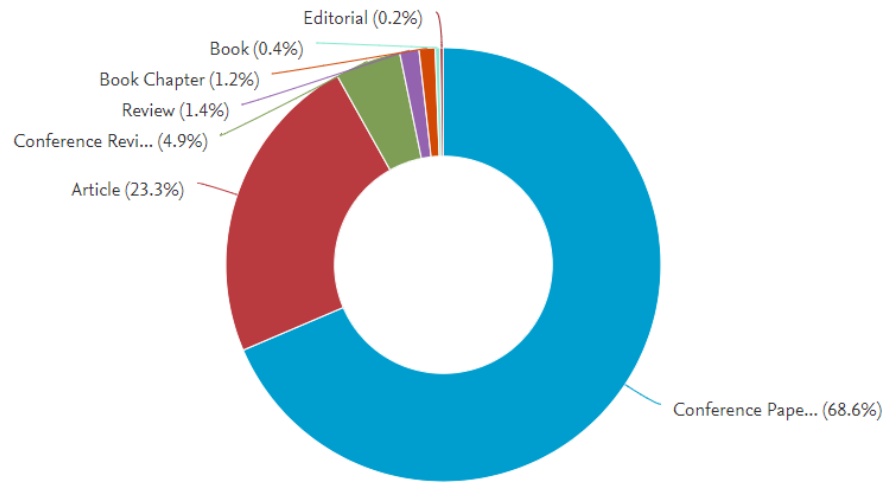

Figure 5. Documents by type Source: Data extracted from Scopus

Having verified the relevance of conferences, it was analyzed in Figure 6 that the events producing the most documents on the subject were the Lecture Notes in Computer Science with evident growth each year since 2015, other prominent conferences were Advances in Intelligent Systems And Computing, ACM International Conference Proceeding Series, Ceur Workshop Proceedings and Communications in Computer And Information Science. 
IADIS International Journal on WWW/Internet

\section{Documents per year by source}

Compare the document counts for up to 10 sources.

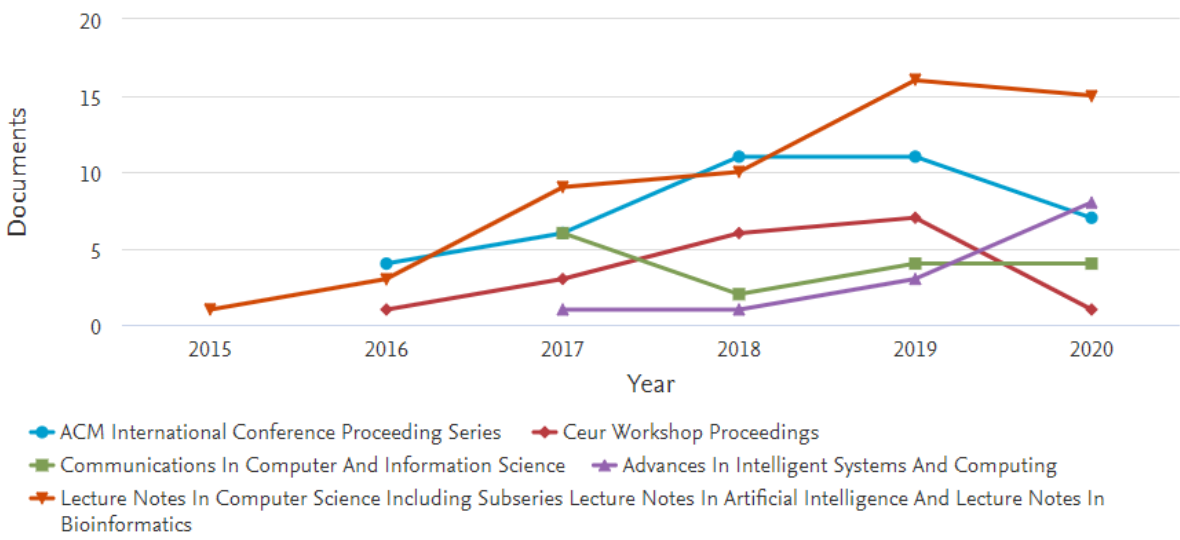

Figure 6. Documents per year by source Source: Data extracted from Scopus

\subsection{Data Presentation and Inter-Relations}

According to Zupic and Cater (2015) one of the essential tasks for advancing a particular line of research is to synthesize the findings of past researches. Using bibliometric techniques to investigate and analyze the scientific literature and its relationships, such relationships will be addressed in this section.

Starting from Scopus' own results analysis tool according to Figure 7, it is possible to identify that USA, Germany, and China are the countries that publish the most on the subject, it is worth pointing out that despite the more representative demographic density in China and USA, Germany still figures in second place, however this analysis is not the focus of this work. In Fig. 7 it is also worth noting that Brazil is the only country in the Southern Hemisphere among the 10 that most publish. 


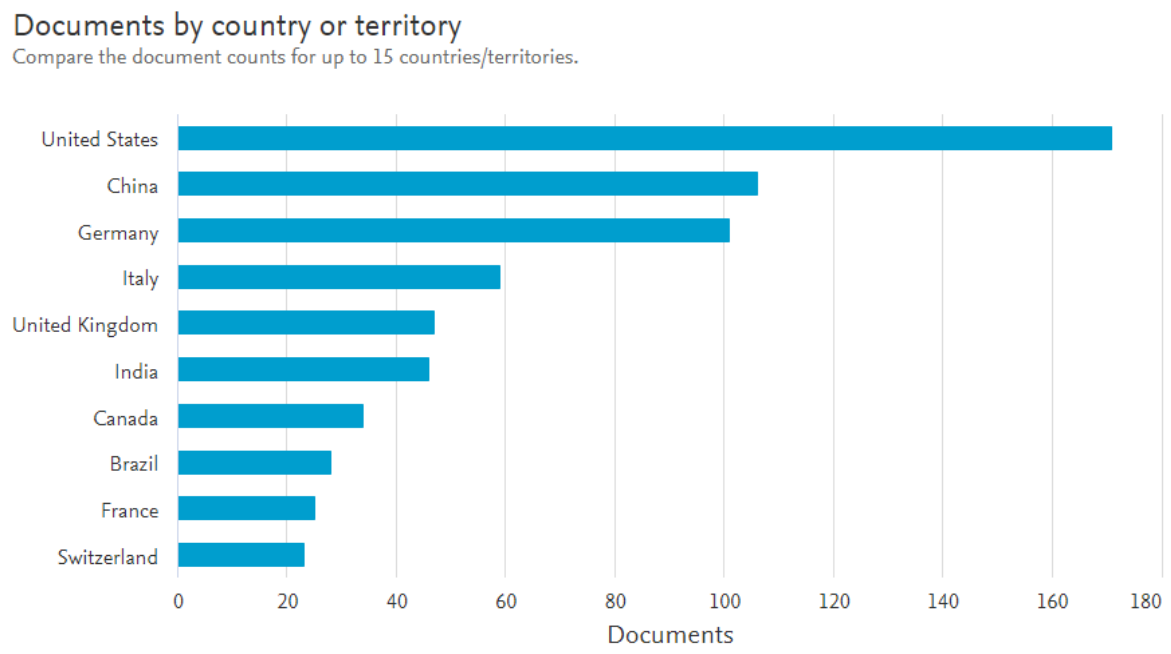

Figure 7. Documents by country or territory Source: Data extracted from Scopus

Analyzing Figure 8 it can be seen that the 3 main supporters of the subject are institutions from the USA, China, and Europe, in direct proportion to the amount of work produced in each of these regions.

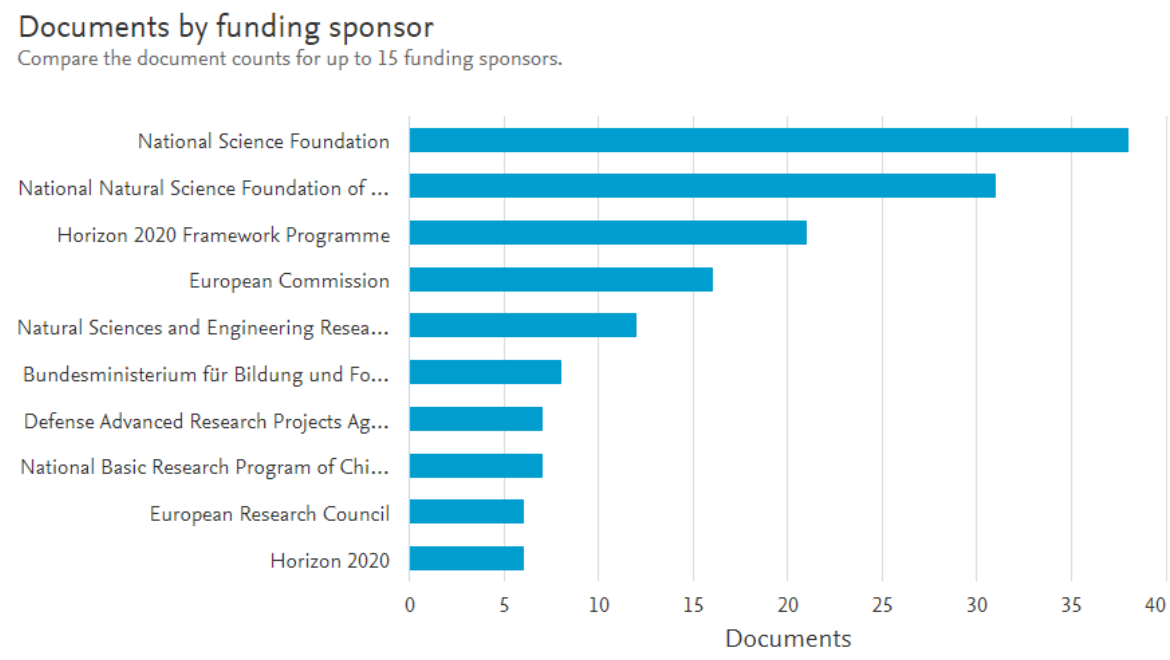

Figure 8. Documents by funding sponsor Source: Data extracted from Scopus 
IADIS International Journal on WWW/Internet

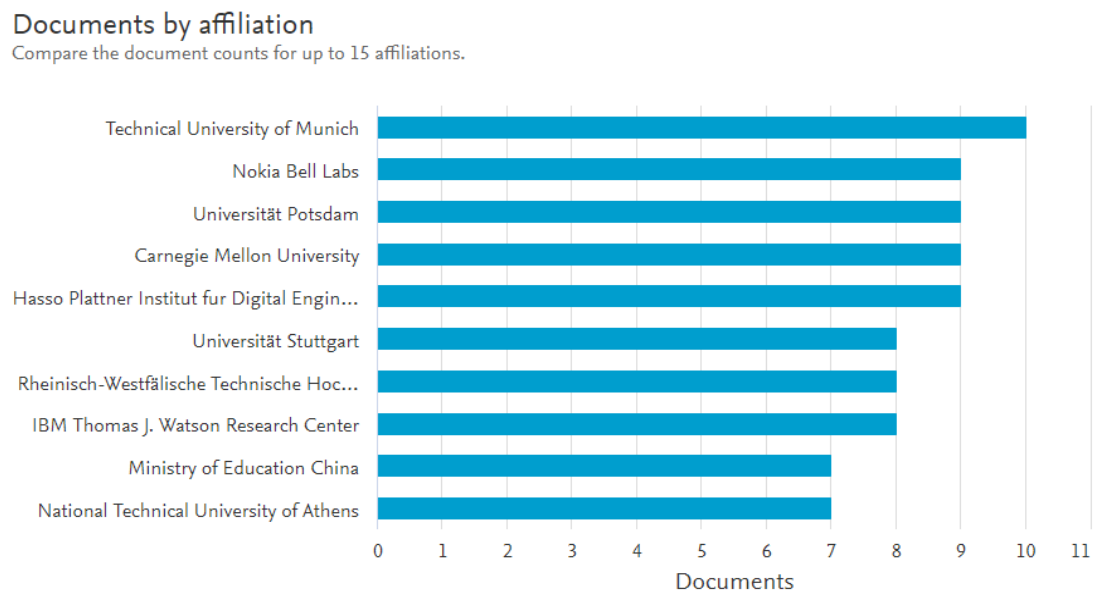

Figure 9. Documents by affiliation

Source: Data extracted from Scopus

As for the affiliated institutions, it is highlighted that the 4 main institutions are German, according to Figure 9, with emphasis on the University of Potsdam, which has as affiliates the authors who have most published in the last 5 years, according to Figure 10: C. Meinel, M. I. H. Sukmana, K. A. Torkura, and F. Cheng who have several works together about cloud, which proves to be an important center of research on issues related to security and microservices.

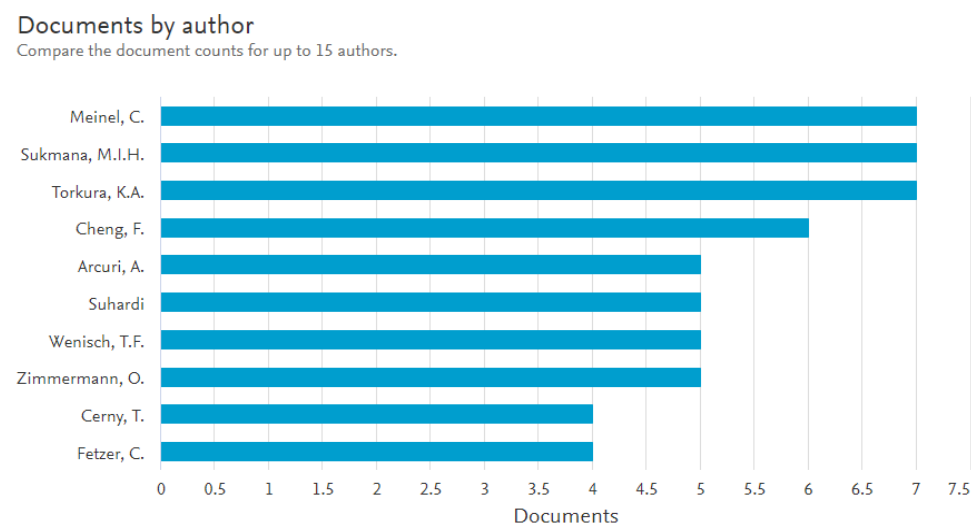

Figure 10. Documents by author

Source: Data extracted from Scopus

Figure 11 presents the co-citation network, according to Zupic and Cater (2015) the probability of similarity of content is higher, the more items are cited together, so the analysis of this network will present the authors who have similar line of research.

It is observed the presence of 6 clusters of authors that are commonly co-cited in academic works, it is possible to validate that there is a great dispersion of data, and the most cited authors, in this order, are M. Fowler, Y. Zhang, S. Newman, R. Buyya, P. Jamshidi and Y. Chen emphasizing the diffusion of this theme by authors from Oceania, Asia, America and Europe, as can also be seen in Figure 12. 


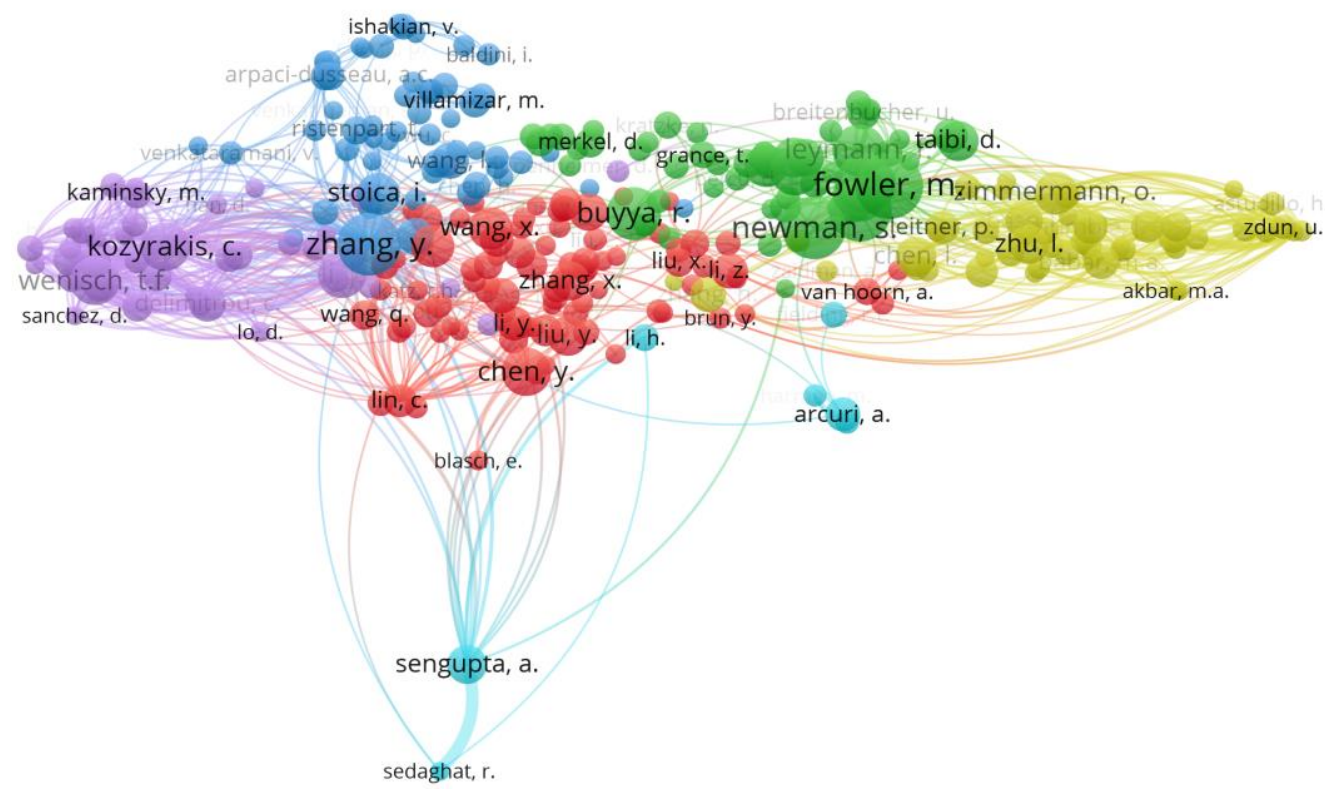

Figure 11. Network diagram of author co-citation Source: Generated by VOSviewer with data extracted from Scopus

As described in section 4.1, Newman was one of those responsible for popularizing the term microservice, so many works start from the definition given by him and complemented by Fowler, showing a high degree of citation of these authors' books in all the clusters presented. The research area led by these authors is in green and can be considered the one that started the others, because it presents the most cited authors and the oldest works of this revision. M. Fowler, S. Newman, R. Buyya, J. Lewis, P. Jamshidi, C. Pahl and F. Leymann, with a total of more than 100 citations each, demonstrates that this cluster has well consolidated authors in the area.

Although there are authors already consolidated in the first cluster, there are 5 other clusters, which indicates that the subject is being approached in different ways, and the distance of the authors from the initial source is growing constantly, generating new clusters with other references and possible new lines of research. A clear example is Y. Zhang, The most cited author of the cluster in blue and also the second most cited of all the works, this cluster presents a departing area, which may represent the creation of a new line of research soon, this can be observed with the authors M. Villamizar and V. Ishakian, who are far away from the center of the cluster, which is strongly linked by the authors Y. Zhang and I. Stoica.

There are also 3 other clusters with great relevance to the subject, by authors C. Kozyrakis and T. Wenisch, represented on the left with the color purple. In the center, with the color red, we can highlight the authors Y. Chen, X. Wang, and Y. Wang as authors more representative of the cluster. And on the right, in the yellow color, there are a large number of authors, without one clear stand out, being the most cited authors O. Zimmermann and L. Zhu. 
IADIS International Journal on WWW/Internet

These clusters represent possible new approaches to the subject, we can clearly notice that the clusters in yellow and blue are the ones with the greatest dispersion within themselves, which indicates that there is a creation of new research lines emerging, with new references. A clear example of this break in clusters can be noticed by the cyan color in the lower part of Figure 11, where there is a cluster that has only 7 authors, being the smallest group and the one that is furthest from the others, clearly indicating a departure from the current lines of research that are being pursued, this cluster is headed by A. Sengupta and A. Arcuri.

In addition to these clear divisions, it is possible to note that the theme continues to expand, endorsing the analysis made in section 4.1 that the number of publications on the theme is on the rise. The distancing of authors such as U. Zdun and V. Ishakian from its current nucleus may represent the creation of a new cluster, demonstrating the continuous growth of the theme and ways to approach it.

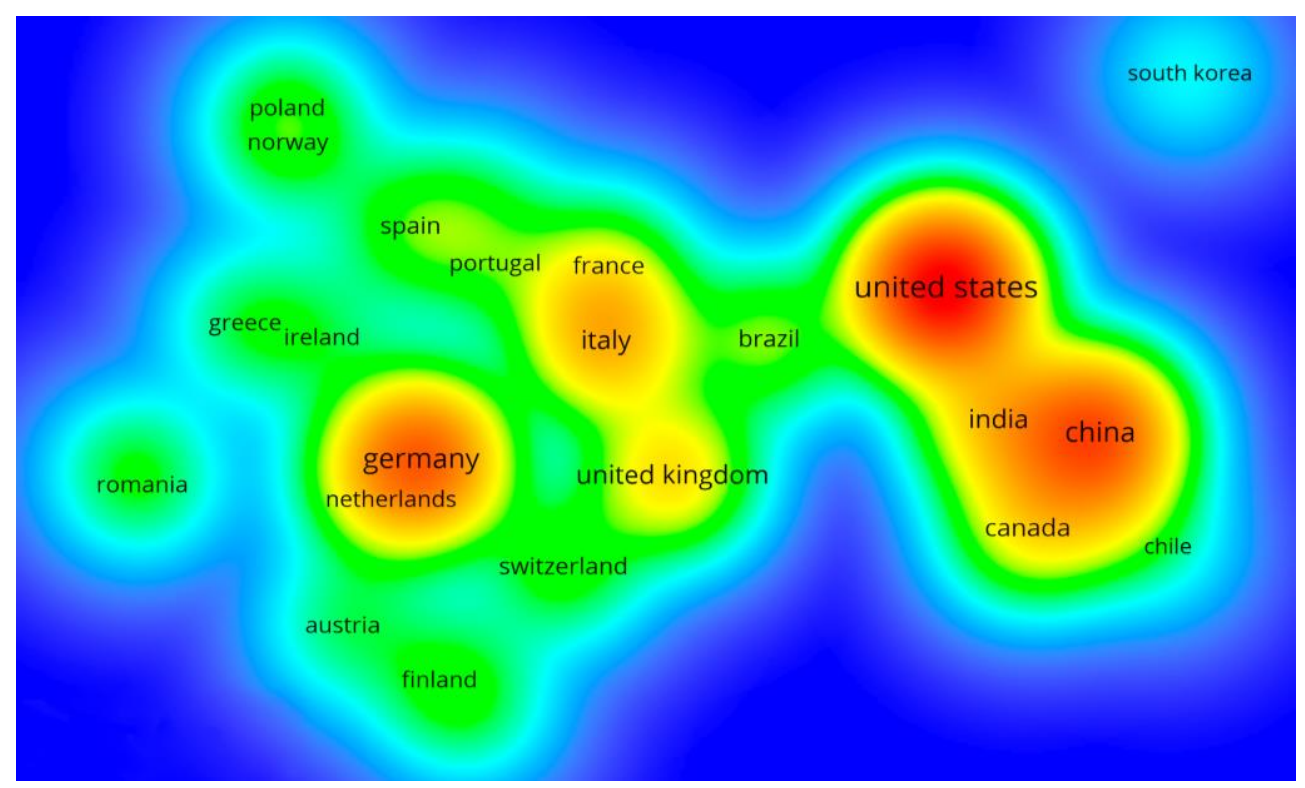

Figure 12. Heatmap diagram of co-authorship by country

Source: Generated by VOSviewer with data extracted from Scopus

About the co-authorship relationship between countries, the data analysis generated the heat map presented in Figure 12 according to the geographical origin of the authors who have publications together, this analysis does not aim to evaluate which countries have the highest number of publications, but rather to evaluate the co-authorship relationship between nations.

Four main clusters of countries with a large co-authorship relationship can be observed, the largest being led by the United States and China, also including India, Australia, Canada and Chile. Next there is Germany with great co-authorship with several other countries in Europe, with Italy and the United Kingdom being its main links, which in turn are connected to the United States cluster by the Brazil cluster, indicating that Brazil is a link between European and North American works. 
The countries that have a centralized position, besides having a great co-authorship relationship with each other, also have a good relationship with the countries of neighboring clusters, which shows the great variety of publications with different countries and may indicate diversity in the lines of research developed. One particularity is South Korea, which is isolated but has some relationship with the USA.

In order to understand which are the research fronts, Figure 13 was used, showing the heat map of bibliographic coupling. The bibliographic coupling uses the number of references shared between two documents to identify similarity between them, the more bibliographic overlapping the more similar the documents tend to be (Zupic and Cater, 20154). Therefore, through this map it is possible to highlight the main research fronts regarding the subject.

For the purpose of this analysis, the last 3 years of publications have been evaluated, because this way it is possible to reveal which are the most consolidated approaches, those that are being strengthened, and also to identify which are emerging and have no citation, besides also excluding lines of research that were initiated and abandoned by the community.

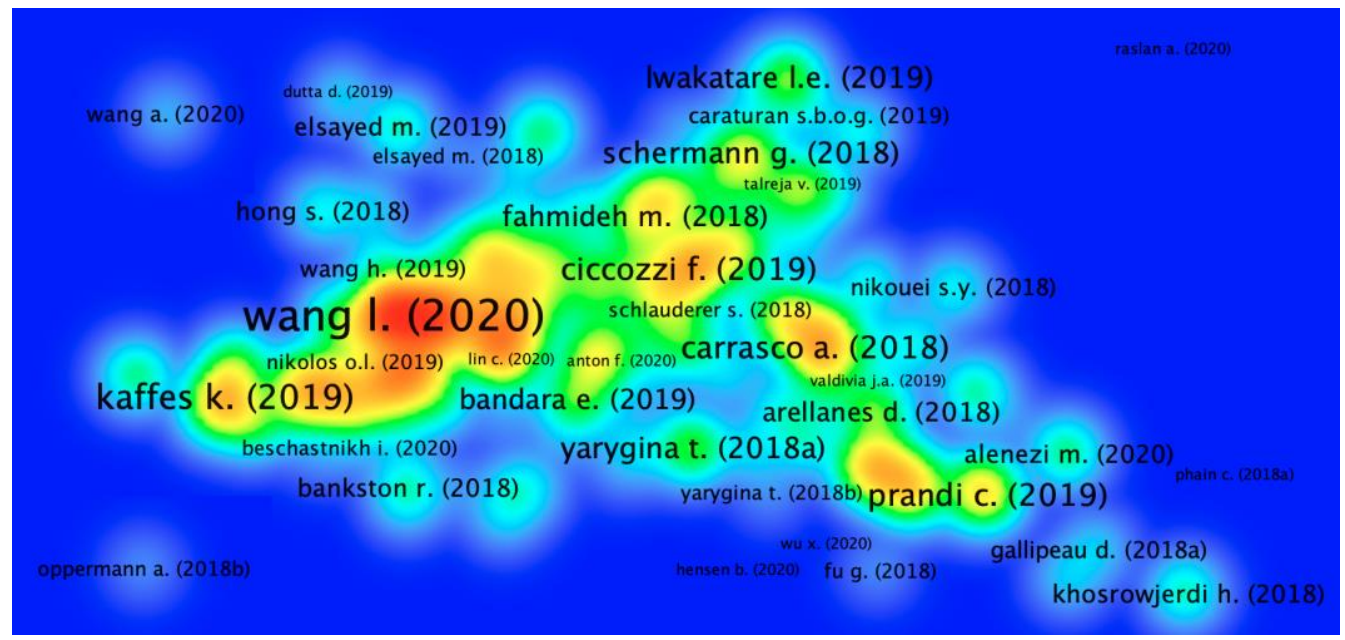

Figure 13. Heatmap of bibliographic coupling of documents Source: Generated by VOSviewer with data extracted from Scopus

The heat map of Figure 13 has a total of 542 documents, a smaller number than the one presented in Figure 11 of co-citation, therefore it does not include all the documents already analyzed. This analysis consolidates the adherence between the works found in the Scopus base, excluding types of publications that are not indexed in scientific bases, such as books, non-scientific articles and others.

Figure 13 shows a total of 17 clusters, the largest of which representing $13.3 \%$ of total documents have high coupling, it was observed that this cluster has a research front related to the serverless computing paradigm. The first highlight to be observed is Wang I. et al with the article "Peeking Behind the Curtains of Serverless Platforms", 2020, specifically addressing how APIs are currently being built, explaining how platforms that offer this service isolate functionality, being containers or virtual machines, while they are running and what security implications should be taken into consideration. 
IADIS International Journal on WWW/Internet

Another work of the same grouping to be highlighted is "Serverless Computing: An Investigation of Factors Influencing Microservice Performance" by Lloyd et al, 2018, whose objective is to evaluate the performance of microservices in relation to the state in which the infrastructure that enables serverless computing is in, highlighting that there are drastic variations in performance according to the state of the infrastructure, proving that serverless computing applications depend directly on the state of the infrastructure for performance measures.

Both articles involve microservice as the most suitable application for these systems, highlighting the gains in availability, horizontal and vertical scalability and short development cycles, enabling cost reduction for application deployment.

The second most representative cluster has $12.2 \%$ of the documents, it has a strong tendency to compare, test and validate the cloud native applications in the most varied ways, since their work distributes common benchmarking measurement techniques among different cloud providers, as described in the work "Benchmarking Heterogeneous Cloud Functions" by Malawski et al, 2017. Papers from Acuri et al, which addresses the EVOMASTER tool for generating evolutionary and automated test cases are also present in this cluster.

In the same cluster there are other articles related to security, among them is the most relevant article of this cluster, by Elsayed et al, "Offering security diagnosis as a service for cloud SaaS applications", 2019, that performs the empirical assessment using the SDaaS solution, which uses benchmarking of other market tools to determine the capacity of detection and evaluation of vulnerabilities, protection against data violation.

Another outstanding work in the same cluster involving security is the "Defense-in-depth and Role Authentication for Microservice Systems" by Jander et al, 2020, which focuses on showing that the protection of HTTP services using TLS standards is not enough to ensure the protection of the application. The approach presented is to use standard cryptographic primitives that can be combined to provide a flexible communication system providing a high level of security.

The third most representative cluster presents $10 \%$ of the articles, with the article "From Monolith to Microservices: A Classification of Refactoring Approaches" by Fritzsch et al (2018) being the most relevant of the cluster, through a systematic review compares systems refactoring processes for their transformation from monoliths into microservices based systems. Another cluster highlight is the work "Functionality-oriented Microservice Extraction Based on Execution Trace Clustering" which also explores the theme of monolith migration to the microservice architecture, comparing 3 methods of extraction of candidate entities for the microservice model with a new methodology proposed to FoME (Functionality-oriented Microservice Extraction) with very expressive results from Jin et al.

The work "Docker-Sec: A Fully Automated Container Security Enhancement Mechanism", one of the most visualized of the cluster, deals with a prevention mechanism of systems based on microservices that use Docker containers. According to this article, the use of containers brings several challenges regarding security, due to "their direct communication with the host kernel, allowing attackers to break into the host system and co-located containers more easily than Virtual Machines" (Loukidis-Andreou et al, 2018) proposing then an automated mechanism called Docker-sec for the creation of rules and configurations in containers in order to protect containers from vulnerabilities with minimal loss of performance.

Representing 9.2\% of the works, Carrasco et al stands out with the work "Migrating towards Microservices: Migration and Architecture Smells" and "Migrating Web Applications from Monolithic Structure to Microservices Architecture" by Ren et al, highlighting the migration 
process to microservices from a traditional architecture (Yarygina and Bagge, 2018), which demonstrates that the architecture that uses microservices is gaining space (Kratzke, 2018). This research front presents comparisons and case studies between traditional architecture and the gains in the use of microservices, beyond the challenges found in this architecture.

Some of the works that present these comparisons from the perspective of the life cycle of the applications, featuring the concept of DevOps, such as the works "DevOps in practice: A multiple case study of five companies" by Lwakatare et al, "An empirical study of architecting for continuous delivery and deployment" by Shahin et al and "Overcoming Challenges With Continuous Integration and Deployment Pipelines: An Experience Report From a Small Company" by V. Debroy and S. Miller.

Another case study to be highlighted is that of Luz et al, with the title "An experience report on the adoption of microservices in three Brazilian government institutions", which highlights the various benefits that the adoption of microservices has brought to the institutions mentioned.

An important relation found in this analysis is the fact that the main works of the third and fourth largest clusters deal with the migration from monoliths to microservices demonstrating that there are several challenges involved in the decision to break a monolithic system, from the design and approaches to choosing candidate entities to become independent services, to techniques, tools and methodologies for system division based on static code analysis, execution tracing or communication.

The fifth cluster represents $8.5 \%$ of the total works, this group has relevant works from the point of view of vertical and horizontal scalability and prediction of failures, however it approaches these mechanisms from the point of view of machine learning to make decisions on how to proceed, the main works found are "Elastic Load Balancing for Dynamic Virtual Machine Reconfiguration Based on Vertical and Horizontal Scaling" by Sotiriadis et al and "Time: Architecture-aware online failure prediction" by Pitakrat et al.

"If Docker is the Answer, What is the Question?" by Zhu et al try to find out for which challenges Docker effectively is the answer to, bringing reflections about the application lifecycle and the architectures that best fit this infrastructure delivery model. This work is part of the cluster that represents $7.4 \%$ of the total work, it tries to understand a little more about the architecture of microservices, security implementations and their challenges. Tsikerdekis et al present approaches to using Honeypot in an intelligent way in "Approaches for Preventing Honeypot Detection and Compromise", presenting some ways to prevent attacks in cloud applications, as well as Li et al in "Exploring New Opportunities to Defeat Low-Rate DDoS Attack in Container-Based Cloud Environment".

A cluster that represents $7.2 \%$ of the analyzed works, has a great predominance of the performance applied in microservices theme, having the most cited document "Seer: Leveraging Big Data to Navigate the Complexity of Performance Debugging in Cloud Microservices" which presents the Seer system for debugging services, from the statement "predictable performance is even more critical as cloud services transition from monolithic designs to microservices" in the work of Gan et al. Another relevant article is "SoftSKU: optimizing server architectures for microservice diversity @ scale" which deals with cloud services performance through server tuning. As well as the articles "Enhancing Server Efficiency in the Face of Killer Microseconds" and "RPCValet: NI-Driven Tail-Aware Balancing of $\mu$ s-Scale RPCs" which also address the issue of performance in microservices by proposing a large increase in multithreading according to the work of Mirhosseini et al. and the use of communication queues in Daglis et al. 
IADIS International Journal on WWW/Internet

The following cluster is distributed in several points of the heat map, presenting some degree of coupling with several clusters, but creating its own, which represents $7 \%$ of the documents, among the works we can highlight the one from Martin et al entitled "Docker ecosystem Vulnerability Analysis", which has some degree of coupling with I. Wang, but is more interested in pointing out the vulnerabilities in an ecosystem based on Docker, presenting real cases of how these weaknesses can be explored and proposing possible solutions, and discussing the adoption of Docker provided in PaaS.

Another work of this cluster that presents relevant discussions about security is by Yarygina et al, where they show in "Overcoming Security Challenges in Microservice Architectures" several vulnerabilities found in microservices, giving details of the threats in the Hardware, Virtualization, Cloud, Communication, Application and Orchestration layers that a microservice has. They also present mitigation for these threats.

There are 9 other research fronts represented in Fig. 13, however each one of them represents about $5 \%$ or less of the total of documents, therefore the large distribution of research fronts indicates that the theme is still in expansion and does not have high consolidated references in all areas of activity, besides showing that some new research clusters are emerging, presenting low bibliographic coupling with the current themes that are currently highlighted.

Among these clusters, it is relevant to highlight that there is a research front that aims to deepen techniques of monitoring and Self Healing of microsservices.

\subsection{Detailing, Integrating Model and Evidence-Based Validation}

One factor that highlights the assertiveness of the data model chosen for this meta-analytical review is finding in the bibliography at least one systematic review article (Mariano and Rocha, 2017). In the research, 14 systematic review articles were found related to the theme investigated Alshuqayran et al. (2016) and Hassan et al. (2020), for example, presented systematic studies on metadata and its main challenges, whose notes helped to direct this study.

Applying the Law of Elitism of Price (1963) we find that on the base of a total of 2548 authors in the area, according to the formula of the $\operatorname{Law} \sqrt{2548}$ the result is 50 authors who would be the elite in the area and who, according to this law, should be responsible for $50 \%$ of publications in the area. However, a total of only 221 articles published by these authors out of the total of 837 observed were found, i.e., about $26 \%$, which could show that there is still no elite formed in the area (Alvarado, 2009).

It was also verified that the $20 \%$ of the authors with the highest work production are responsible for about one third of the articles among all the analyzed authors, that is, they do not represent a domain regarding the quantity of documents produced. Still regarding production, only 337 authors out of 2548 have 2 or more articles related to the subject. However, analyzing the number of citations, the 50 authors with the most citations represent $50.47 \%$ of all the citations in the area, with 1050 authors having no citations and 417 having only 1 citation showing that the most relevant documents are concentrated in an elite of authors whose detailing was done in Section 4.2. 


\section{CONCLUSION}

From the studies carried out following the Theory of the Consolidated Meta-Analytic Approach, it can be verified that far more than just the relevance of the security challenges applied to the architecture of microservices, this is a theme that still brings great opportunities for research and deeper study considering the complexity involved in architectures of autonomous microsystems distributed in a network.

The methodology used based on stages was also a great facilitator in the systematization of the metadata found in the analyzed scientific bases, with Scopus standing out. Thus, it was identified that TEMAC can be a great ally of researchers (Cerqueira et al., 2020) who seek to analyze more relevant keywords, main authors and research centers, or even apply bibliometric techniques in order to identify relevant inter-relations and trends.

The research model used on scientific databases can also be used as a basis for other studies as a starting point or as a model to review or validate a research.

Some of the main findings were that the most influential authors are M. Fowler, Y. Zhang, S. Newman, R. Buyya, P. Jamshidi, and Y. Chen, the countries that have most published on the subject are China, USA and Germany and there is great opportunity for research on the subject.

Regarding the approaches found in the literature, it was noticed that several outstanding works are related to the challenges of using secure microservices, therefore several articles present tools and framework creations for the separation of monolithic systems and design of microservices, monitoring, tuning and configuration tools, with predominance of automated solutions. There is also a large number of papers that present and explore threats, besides providing means to mitigate the risks presented by attacks or system failures.

Thus, according to Alshuqayran et al. (2016), the greatest challenges presented are related to communication/integration between services, performance, fault tolerance, security, log and monitoring and deployment operations, it was verified in section 4.2 that most of the work seeks to answer some of these topics.

In the future this study can be expanded using more databases such as Microsoft Academy, or comparing Scopus data with Google Scholar and WoS results, and also including the Internet of Things subarea which has shown great repercussion in literature (Washizaki et al., 2020).

\section{ACKNOWLEDGEMENT}

The authors would like to thank the support of the Office of Institutional Security of the Presidency of the Republic (TED 002/2017). 
IADIS International Journal on WWW/Internet

\section{REFERENCES}

A. Mirhosseini, A. Sriraman and T. F. Wenisch, (2019). "Enhancing Server Efficiency in the Face of Killer Microseconds," 2019 IEEE International Symposium on High Performance Computer Architecture (HPCA), Washington, DC, USA, 2019, pp. 185-198., doi: 10.1109/HPCA.2019.00037.

Akshitha Sriraman, Abhishek Dhanotia, and Thomas F. Wenisch. (2019). SoftSKU: optimizing server architectures for microservice diversity @ scale. In Proceedings of the 46th International Symposium on Computer Architecture (ISCA '19). Association for Computing Machinery, New York, NY, USA, 513-526. doi: 10.1145/3307650.3322227

Alexandros Daglis, Mark Sutherland, and Babak Falsafi. (2019). RPCValet: NI-Driven Tail-Aware Balancing of $\mu$ s-Scale RPCs. In Proceedings of the Twenty-Fourth International Conference on Architectural Support for Programming Languages and Operating Systems (ASPLOS '19). Association for Computing Machinery, New York, NY, USA, 35-48. doi: 10.1145/3297858.3304070

Alvarado, Rubén Urbizagástegui. (2009). Elitism in the literature on the productivity of authors [Elitismo na literatura sobre a produtividade dos autores]. Ciência da Informação, 38(2), 69-79. doi: 10.1590/S0100-19652009000200006

Alshuqayran, N.; Ali, N.; Evans, R. (2016). "A systematic mapping study in microservice architecture" Proceedings - IEEE 9th International Conference on Service-Oriented Computing and Applications, SOCA

Beuren, I. M.; Souza, J. C. d. (2008). "In search of a proposal outline for the classification of international accounting journals for Qualis CAPES [Em busca de um delineamento de proposta para classificação dos periódicos internacionais de contabilidade para o Qualis CAPES]", Revista Contabilidade \& Finanças, vol.19, n.46, pp.44-58. doi: 10.1590/S1519-70772008000100005.

Cerqueira, J. A. S. d. et al. (2020). "Exploratory Overview on Breaking CAPTCHAs Using the Theory of the Consolidated Meta-Analytic Approach," 15th Iberian Conference on Information Systems and Technologies (CISTI), Sevilla, Spain, pp. 1-6. doi: 10.23919/CISTI49556.2020.9140983.

Chondamrongkul, N.; Sun J.; Warren, I. (2020). "Automated Security Analysis for Microservice Architecture," IEEE International Conference on Software Architecture Companion (ICSA-C), Salvador, Brazil, pp. 79-82. doi: 10.1109/ICSA-C50368.2020.00024.

Fritzsch, J., Bogner, J., Zimmermann, A., \& Wagner, S. (2019). From Monolith to Microservices: A Classification of Refactoring Approaches. Lecture Notes in Computer Science, 128-141. doi: 10.1007/978-3-030-06019-0_10.

Guedes, V. L. S.; Borschiver, S. (2005). "Bibliometrics: a statistical tool for the management of information and knowledge, in information, communication and scientific and technological evaluation systems [Bibliometria: uma ferramenta estatística para a gestão da informação e do conhecimento, em sistemas de informação, de comunicação e de avaliação científica e tecnológica]", Encontro Nacional de Ciências da Informação, 6., Salvador/BA.

Hassan, S.; Bahsoon, R.; Kazman, R. (2020). "Microservice transition and its granularity problem: A systematic mapping study". Softw Pract Exper. 50: 1651- 1681. doi: 10.1002/spe.2869

Jin, W., Liu, T., Zheng, Q., Cui, D., \& Cai, Y. (2018). Functionality-Oriented Microservice Extraction Based on Execution Trace Clustering. 2018 IEEE International Conference on Web Services (ICWS). doi: 10.1109/icws.2018.00034

Kratzke, N. (2018). "A Brief History of Cloud Application Architectures", Appl. Sci. 8, 1368. doi: 10.3390/app8081368.

Lewis, J. and Fowler, M. (2014). Microservices [Online]. Available at: http://martinfowler.com/articles/microservices.html. (Accessed: 23 Nov 2020) 
Loukidis-Andreou, F., Giannakopoulos, I., Doka, K., \& Koziris, N. (2018). Docker-Sec: A Fully Automated Container Security Enhancement Mechanism. 2018 IEEE 38th International Conference on Distributed Computing Systems (ICDCS). doi: 10.1109/icdcs.2018.00169

Mariano, A. S.; Rocha, M. (2017). "Literature review: Presentation of an integrative approach [Revisão da literatura: Apresentação de uma abordagem integradora]," XXVI Congreso Internacional de la Academia Europea de Dirección y Economía de la Empresa (AEDEM), Reggio Calabria, vol. 26.

Newman, S. (2015). "Building Microservices", O’Reilly Media.

Perdigão, A.; Prado, F.; Mariano, A. S. (2019). "Use of scientific database: an exploratory study using the Consolidated Analytical Meta Approach Theory - TEMAC [Uso de base de dados científica: um estudo exploratório por meio da Teoria do Enfoque Meta Analítico Consolidado -TEMAC]".

Price, J. D. d. S. (1963). "Little science, big science", New York: Columbia University Press.

Rovira, C.; Codina, L.; Guerrero-Solé, F.; Lopezosa, C. (2019). "Ranking by Relevance and Citation Counts, a Comparative Study: Google Scholar, Microsoft Academic, WoS and Scopus", Future Internet. doi: 10.3390/fi11090202.

Washizaki, H.; Ogata, S.; Hazeyama, A.; Okubo, T.; Fernandez, E. B. ; Yoshioka, N. (2020). "Landscape of Architecture and Design Patterns for IoT Systems," in IEEE Internet of Things Journal. doi: 10.1109/JIOT.2020.3003528.

Yarygina, T.; Bagge, A. H. (2018). "Overcoming Security Challenges in Microservice Architectures," IEEE Symposium on Service-Oriented System Engineering (SOSE), Bamberg, pp. 11-20. doi: 10.1109/SOSE.2018.00011.

Yu Gan, Yanqi Zhang, Kelvin Hu, Dailun Cheng, Yuan He, Meghna Pancholi, and Christina Delimitrou. (2019). Seer: Leveraging Big Data to Navigate the Complexity of Performance Debugging in Cloud Microservices. In Proceedings of the Twenty-Fourth International Conference on Architectural Support for Programming Languages and Operating Systems (ASPLOS '19). Association for Computing Machinery, New York, NY, USA, 19-33. doi: 10.1145/3297858.3304004

Zupic, I. and Čater, T. (2015). 'Bibliometric Methods in Management and Organization', Organizational Research Methods, 18(3), pp. 429-472. doi: 10.1177/1094428114562629. 